Schmerzhypothese zur Erklärung des Galvanotropismus annehmen können, verstehen wir nicht.

Zum Schlusse wollen wir darauf hinweisen, dass man eine Zeit lang auch geneigt war, die Reaktionen der Thiere bei Verletzung der Ampullen auf Schmerzempfindungen zurückzuftuhren. Davon ist man mit Recht zurückgekommen. Ohne die Möglichkeit einer schmerzhaften Wirkung des Stromes leugnen zu wollen sind wir der Meinung, dass die galvanotropischen Erscheinungen mit demselben Rechte aus einer Wirkung des Stromes auf die Spannung und Energieentwicklung der Locomotionsmuskeln abzuleiten sind, wie die Zwangsbewegungen der Thiere auf einer Centrifugalmaschine oder nach Verletzung des Ohres oder des Gehirns.

Das Resultat unserer Versuche lässt sich in folgenden Satz zusammenfassen: Die galvanotropischen Erscheinungen bei Wirbelthieren beruhen (wie bei Krebsen) darauf, dass der constante Strom gleichsinnige Aenderungen der Spannung und Energieentwicklnng assoziirter Muskelgruppen herbeifuihrt, wodurch die Bewegung gegen einen Pol erleichert, die Bewegung gegen den entgegengesetzten Pol erschwert wird.

\title{
Die polare Erregung der lebendigen Substanz durch den constanten Strom.
}

IV. Mittheilnng.

Von

Prof. Max Verworn, Jena.

Hierzu Tafel IV und 1 Textfigur.

In meiner zweiten Mittheilung iber die polaren Wirkungen des constanten Stromes auf die lebendige Zelle ${ }^{1}$ ) hatte ich unter anderem auch ïber die Erscheinungen berichtet, welche sich bei galvanischer Reizung an verschiedenen Amoebenformen beobachten lassen. Da ich im April dieses Jahres zufällig in den Besitz

1) M. Verworn: „Die polare Erregung der Protisten durch den galvanischen Strom". In diesem Archiv Bd. 46, 1889. 
ganz aussergewöhnlich grosser Amoeben gekommen bin, so habe ich die Gelegenheit, die mir das ausgezeichnete Material bot, benutzt und daran meine Untersuchungen weiter fortgesetzt, so dass ich nunmehr in der Lage bin, meine früheren Mittheilungen wesentlich zu ergänzen und vor allem die Deutung der polaren Wirkungen des constanten Stromes am Amoebenkörper mit aller wiinschenswerthen Klarheit zu liefern.

\section{Amoeba proteus.}

Die von mir benutzte Amoebenform, die ich in grösserer Menge in dem Wasser eines Grabens am Luftschiff bei Jena fand, besitzt die typischen Charaktere der gewöhnlichen Am o eba prote us. Ein sehr grobkörniges, mit allerlei fremden Inhaltsbestandtheilen durchmischtes Endoplasma enthält den in der Einzahl vorhandenen runden Zellkern, der nur durch Färbungsmittel deutlich sichtbar zu machen ist, sowie eine grosse contractile Vacuole. Das hyaline Exoplasma, das beim Kriechen an der Oberfläche der stumpfen Pseudopodien bald bierhin, bald dorthin vorfliesst, erscheint in ungestörtem Zustande völlig homogen und zeigt keine Wabenstructur. Die Pseudopodien werden in breiter Lappen- oder Fingerform nach verschiedenen Richtungen hin ausgestreckt. Oft ist die ganze Oberfläche des klumpigen Körpers von kurzen fingerförmigen Pseudopodien rings herum besetzt, so dass eine Form wie $\mathrm{Amoeba}$ p o l y podia herauskommt (Taf. IV, 1), oft ist die ganze Protoplasmamasse zu einem langen Strang ausgestreckt, so dass die Amoebe wurmförmig erscheint (Taf. IV, 2). Die Grösse der Individuen, die ich hatte, betrug im kuglig contrahirten Zustande derselben etwa $0,15 \mathrm{~mm}$ im Durchmesser, im langausgestreckten Zustande des Körpers $0,4-0,5 \mathrm{~mm}$.

Ein einzelnes Individuum dieser A m o e ben wurde jedesmal mit einem Capillarröhrchen isolirt und mit etwas Wasser zwischen die parallelen Thonleisten des electrischen Objectträger-Kästchens ${ }^{1}$ ) gebracht, wo es alsbald nach verschiedenen Richtungen hin seine Pseudopodien auszustrecken begann, indem es nur ganz locker mittels eines feinen schleimigen Secrets an der Unterlage haftete. Wurde dann der constante Strom geschlossen, so trat sofort an der Ka-

1) Vergl. dieses Arch. Bd. 45 . 
thodenseite ein breites, hyalines Pseudopodium aus dem Körper hervor, das sich länger und länger ausstreckte. Die Körnchen strömten aus allen andern Gegenden des Körpers nach diesem Psendopodium zusammen, so dass bald alle ubrigen Pseươpodien eingezogen waren und die ganze Amoebe genau wie A moeba l i max ein einziges grosses keulenförmiges Pseudopodium bildete (Taf. IV, 3), in dem ein axialer Körnerstrom nach der Kathode hinlief, um hier springbrunnenartig an der Peripherie nach hinten umzubiegen. Die Anodenseite dagegen zog sich mehr und mehr zusammen, wurde immer schmaler, und bildete schliesslich einen höckerigen, unregelmässig contourirten Sehwanz. In dieser Keulenform der A moeba lima kroch die Amoebe genau in der Richtung von der Anode nach der Kathode hiniuber, ohne auch nu.r ein Pseudopodium nach einer anderen Richtung auszusenden, solange dor. Strom geschlossen blieb. Die Amoebe war also ausgezeichnet galvanotropisch nach der Kathode. Wurde der Strom geöffnet, so blieb die Körnchenströmung kurze Zeit stehen, Alsbald aber bildeten sich nach dieser und nach jener Richtung hin wieder neue Pseudopodien und nach einiger Zeit hatte die Amoebe wieder die frühere Gestalt und Bewegungsart angenommen. Das waren die Erscheinungen, die sofort pei jeder Beobachtung eines Individuums unter dem Mikroskop in die Augen sprangen.

Zeigte schon die oberflächliche Betrachtung deutlich genug, dass die Wirkung bei der Schliessung und Dauer des constanten Stromes in einer starken expansorischen Erregung an der Kathode und einer contractorischen Erregung an der Anode besteht, so bestätigte ein genaueres Studium der Einzelheiten dieses Ergebniss nur noch mehr. Ich unterzog dabei jeden der beiden Körperpole einzeln einer eingehenderen Untersuchung.

Um die expansorische Erregung an der Kathode noch zweifelloser hervortreten zu lassen, sorgte ich dafür, dass das Protoplasma hier im Moment der Schliessung sich in Contraction befand. Wenn man eine Amoebe in dem Objectträger-Kästchen durch anhaltendes Schiitteln zur kugligen Contraction gebracht hat, dann davert es eine ganze Weile, bis sie wieder beginnt langsam und allmählich neue Pseudopodien hervorzustrecken. Wird nun durch eine Amoebe, die sich soeben vollständig kuglig contrahirt hat, plötzlich ein constanter Strom hindurcbgeschickt, so bricht fast im selben Moment an der Kathodenseite ein breites Pseudopodium 
hervor, das sich mehr und mehr streckt und schliesslich die ganze Protoplasmamasse in sich aufnimmt, so dass nun wieder die für das galvanotropische Kriechen so charakteristische "Limaxform" entsteht. Ausnahmslos wird so die contractorische Erregung, die sonst längere Zeit andauert, an der Kathode durch eine starke expansorische Erregung verdrängt. Das Gleiche zeigt sich, wenn man den Strom, nachdem er einige Zeit durch die Amoebe hindurchgegangen ist, plötzlich wendet. Im Moment der Wendung sieht man die Körnchenströmung und die Bewegung der Amoebe stillstehen. Gleich darauf bricht an der contrahirten Seite d. h. der früheren Anode und nunmehrigen Kathode ein breites hyalines Pseudopodium vor (Taf. IV, 4 a u. b). Dabei ist es bemerkenswerth, dass die contrahirten und fester gewordenen Protoplasmatheilchen selbst nicht sofort wieder in vorquellendes Hyaloplasma zurückverwandelt werden, sondern dass zwischen und neben ihnen neues hyalines Protoplasma hervorquillt, während die contrahirten Massen selbst in das körnige Endoplasma mithineingezogen werden. Nehr und mehr hyalines Protoplasma breitet sich inzwischen vom Innern her nachströmend an der neuen Kathode aus and bald hat die Amoebe wieder die charakteristische Keulenform, in der sie in gleicher Weise wie vorher nach der entgegengesetzten Seite hinkriecht. Also auch hier tritt an der Stelle, wo eben noch eine contractorische Erregung bestand, jetzt, nachdem die Seite zur Kathode geworden ist, expansorische Erregung auf. Diesen Versuch kann man mit ganz dem gleichen Ergebniss beliebig oft wiederholen. Wir habenalsoander Amoebe einen überalle Zweifel erhabenen Fall von expansorischer SchliessungsErregungander Kathode.

Die Erscheinungen an der Anode sind charakterisirt durch das Einschrumpfen des Protoplasmas, das gleichzeitig einen unebenen Contour bekommt und eine Trübung erfährt. Obwohl sich diese Erscheinungen schon ohne weiteres als Contractionserscheinungen documentiren, prüfte ich sie dennoch genauer mit stärkeren Vergrösserungen. Zu diesem Zweek verwandte ich, um ein Immersionssystem benutzen zu können, statt der Thonleisten-Electroden zwei Streifen von Fliesspapier, die einander parallel gegenüber lagen. Zwischen beide wurde der Tropfen mit der Amoebe gebracht, und dann ein Deckglas aufgelegt. Die Ausführung des Versuchs in dieser Form erforderte zwar etwas Geduld, weil das Object beim 
Die polare Erregung der lebendigen Substanz durch den constanten Strom.

Auflegen des Deckglases leicht wegschwamm und die Papierstreifen häufig verschoben wurden. Dennoch aber bot sie mir die Möglichkeit mit einem apochromatischen Immersionssystem (Zeiss 2,0 $\mathrm{mm}$, Apert. 1,30) die feineren Veränderungen am anodischen Pol zu verfolgen und namentlich die Ursache der Trübung des Protoplasmas aufzudecken. Um diese Verhältnisse alle recht deutlich beobachten zu können, sorgte ich nun umgekehrt wie vorher dafür, dass an dem Pol, den ich beobachtete, expansorische Erregung bestand, so dass sich hier eine breite Kuppe von hyalinem Protoplasma befand. Ich schloss alsdann den Strom und stellte den kathodischen Pol, an dem sich das hyaline Protoplasma ausbreitete, ins Gesichtsfeld ein. Wie bereits bemerkt, ist das hyaline Protoplasma der Amoebe in ungestörtem Zustande vollkommen homogen und ohne Wabenstructur. Wurde nun, wenn ein breiter hyaliner Lappen im Gesichtsfelde war, der Strom gewendet, so dass dieser Körperpol plötzlich zur Anode wurde, so stand die Ausbreitung momentan still. Darauf begannen allmählich zahllose, kleine, blasse Vacuolen in dem hyalinen Protoplasma aufzutreten, das, während es mehr und mehr schaumige Structur annahm, sich langsam zusammenzog und von der Anode fort mit dem ganzen Körper nach der Kathodenseite hingezogen wurde. Dabei nahm die Oberfläche der zusammensehrumpfenden Protoplasmamasse ein zerfranztes Aussehen an und erschien bald ganz höckerig (Taf. IV,5). Diese Erscheinungen kamen namentlich bei starken Strömen sehr deutlich zur Entwicklung. Ich habe vor einiger Zeit an anderen Rhizopoden mit vollkommen hyalinen Pseudopodien gezeigt, wie das Wabigwerden des vorher ganz homogenen Hyaloplasmas eine typische contractorische Erregungserscheinung vorstellt ${ }^{1}$ ). So liefern denn auch diese feineren Vorgänge am anodischen Körperpol der Amoebe mit aller nur wünschbaren Klarheit den Beweis, das s wir hier einen deutlichen Fall ron contractorischer Schliessungserregung an der Anode vor uns haben.

Das Verhalten dieser Amoeben bei Schliessung des Stromes, das Vorbrechen des hyalinen Protoplasmas an der Kathode, das Zusammenschrumpfen des anodischen Endes zu einem höckerigen, wabigen Zipfel zeigt so klar und unmittelbar die expansorische

1) M. Verworn: „Der körnige Zerfall. Ein Beitrag zur Physiologie des Todes". In diesem Arch. Bd. 63, 1896. 
Erregung an der Kathode und die contractorische Erregung an der Anode, dass selbst ein Zweifler dureh diesen Anblick sofort davon überzeugt sein muss. Der Mechanismus der galvanotropischen Bewegung ist ebenfalls aus der blossen Beobachtung dieses Verhaltens ohne weiteres klar. Beide Momente, die Contraction am anodischen Pol und die Expansion am kathodischen wirken in demselben Sinne auf die Richtung der Bewegung. Die erstere bewirkt eine Ausbreitung des Protoplasmas, ein Hinkriechen nach der Kathode, die letztere ein Zurückziehen, ein Fortfliessen des Protoplasmas von der Anode. Damit ist der Amoebe die Bewegungsrichtung vorgeschrieben und man kann sie durch Aenderung der Stromesrichtung hinlenken, wohin man sie haben will.

Hat man längere Zeit an demselben Individuum Versuche gemacht, ist der Strom lange geschlossen gewesen, so zeigt die Amoebe nach der Oeffnung beträcbtliche Zeit hindurch einen $\mathrm{Zu}$ stand herabgesetzter Beweglichkeit. Sie bildet in der Regel einen flachen Körper mit kurzen Pseudopodien und verharrt in dieser Form ziemlich lange, oft eine Stunde und mehr (Taf. IV, 6). Allmählich stellt sich dann die normale Beweglichkeit und Form wieder her.

Ein paar Worte möchte ich schliesslich noch über die Form der Amoeben hinzufïgen. Ich habe erwähnt, dass die Form der Amoeba proteus schon ohne dass man absichtlich von aussen her auf sie einwirkt, zwischen zwei Extremen, der Polypodiaform und der Wurmform variirt (Taf. IV, 1 u. 2) und habe ferner gezeigt, wie sie unter dem Einfluss des constanten Stromes cine sehr charakteristische Gestalt annimmt, die Aehnlichkeit hat mit der Limaxform (Taf. IV, 3 u. 4). Diese Thatsachen müssen die Frage nahe legen, ob nicht die verschiedenen "Amoebenarten", die lediglich durch ihre Form, speciell durch die Form ihrer Pseudopodien charakterisirt sind, nur Formvarietäten derselben Amoebenart sind, welche durch die Einwirkung äusserer Factoren bedingt sind. Ohne hier diese Frage in ihrer Allgemeinheit entscheiden za wollen, möchte ich doch einige weitere Thatsachen anführen, die zweifellos in diesem Sinne sprechen.

Als einige der am besten charakterisirten "Amoebenarten" sind bekannt die Amoeba proteus mit ibren stumpfen, lappenförmigen, bald hierbin, bald dorthin vorfliessenden Pseudopodien, die Amoeba limax mit ihrem langgestreckten, ein einziges 
Die polare Erregung der lebendigen Substanz durch den constanten Strom. 53

stumpfes Pseudopodium bildenden Zellkörper und die Amoeba radiosa, deren spitze, stachelförmige Pseudopodien von dem kleinen Zellkörper radiär nacb allen Richtungen hin abstehen. Bei einer Versuchsreihe, die ich im Frübling dieses Jahres an einer im Zimmer gezïchteten Cultur von A moeba li max machte, ergab sich nun, dass ein und dieselbe Amoebe in allen drei Formen erscheinen kann, je nach den äusseren Bedingungen, die man willkürlich bestimmt. In meinem Culturgefäss hatten sich am Oberflächenhäutchen zahllose Schaaren der kleinen Amoeba limax entwickelt, so dass in jedem mikroskopischen Präparat tausende von Individuen unter dem Deckglas waren. Wenn diese Amoeben durch das Uebertragen auf den Objectträger mechanisch gereizt waren, so hatten sie sämmtlich Kugelform, d. h. sie befanden sich im Contractionsstadium (a). Nach einiger Zeit begannen aber nach verschiedenen Richtungen bin stumpfe, lappenförmige Pseudopodien

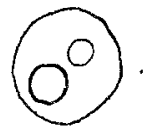

$\alpha$
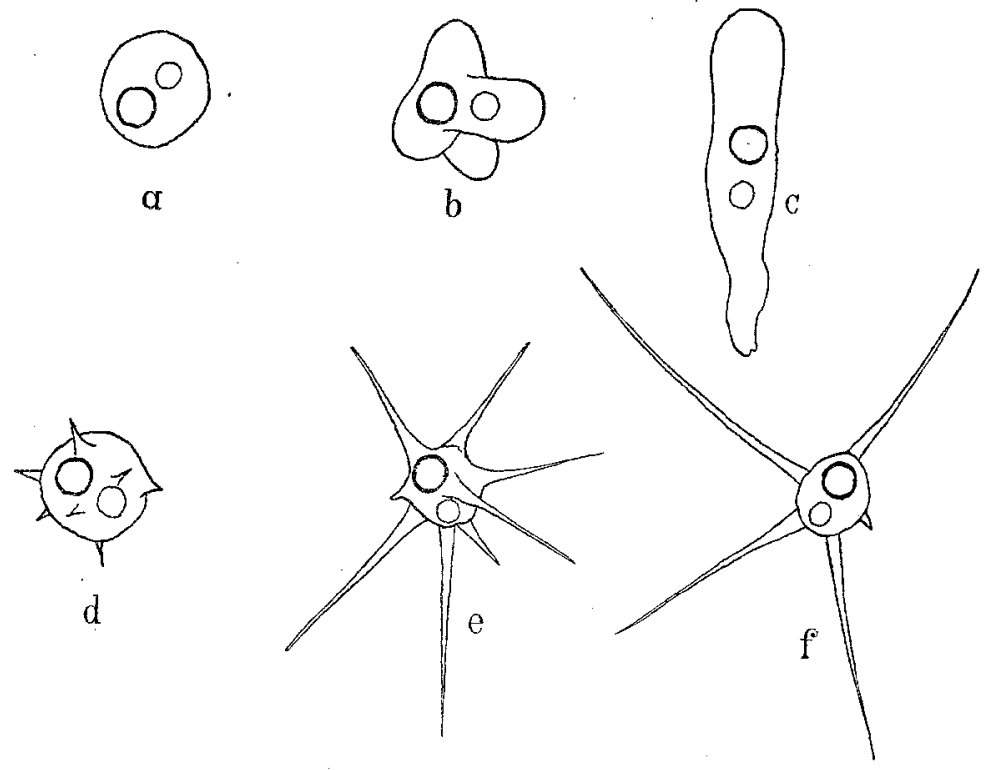

$d$

A moeba limax.

$a$ Kugelform. $b$ Form der Amoeba proteus. $c$ Form der Amoeba limax. $d$ Uebergang zur Form der Amoeba radiosa beim Beginn der KHO-Wirkung. $e$ und $f$ fertige Radiosaformen während der Dauer der KHO-Wirkung.

hervorzutreten und die Amoeben krochen nun längere Zeit in der Form der A moeba prote us umher, indem sie bald hierhin, bald dorthin Pseudopodien entwickelten $(b)$. Nach 10-20 Minuten, 
bei einzelnen Individuen noch später, bildete sich allmählich eine bestimmte Kriechrichtung heraus, d. h. jede Amoebe liess ihre Substanz nunmehr nur nach einer einzigen Richtung vorfliessen, so dass der ganze Körper gewissermaassen ein einziges langgestrecktes Pseudopodium vorstellte, mit anderen Worten die typische Form der A mo eba lim ax annahm, um diese dauernd zu behalten (c). Was mir aber am interessantesten war, das war die Umwandlung in die A moeba radiosa. Machte ich nämlich das Wasser unter dem Deckglas ganz schwach alkalisch durch Zusatz einer ausserordentlich dünnen Lösung von Kalihydrat, so dass es nur eben deutlich alkalisch reagirte, so zogen sich zunächst sämmtliche Amoeben kuglig zusammen und verharrten etwa 10-20 Minuten in dieser Form. Dann aber begannen an der Oberfläche der Kugeln feine Spitzchen hervorzutreten $(d)$, die sich streckten und zu langen, dünnen stachelförmigen Pseudopodien verlängerten, bis die Amoeben sämmtlich nach etwa 20-30 Minuten die typische Form der $\mathrm{A} \mathrm{m}$ o e b a ra d i $0 \mathrm{~s}$ a angenommen hatten $(e, f)$. In dieser Form verharrten sie, solange ich sie vor dem Eintrocknen schützte. Ich habe sie etwa drei Stunden lang verfolgt. Immer behielten sie diese sehr charakteristische Form bei, nur zogen sie hier ein Pseudopodium langsam und träge ein, um dort ebenso langsam und träge ein neves zu entwickeln etc. Wurde das alkalische Wasser durch frisches Wasser ersetzt, so nahmen die Amoeben im Laufe einer halben Stunde wieder ihre gewöhnliche Limaxform an. Der Versuch konnte an denselben Individuen und an jedem neuen Präparat beliebig oft wiederholt werden und lieferte stets den gleichen Erfolg. Versuche mit Säuren, speziell mit verdiunnter Schwefelsäure und Salzsäure führten nur zu dem Resultat, dass sich die Amoeben bei einer Concentration, die überhaupt wirksam ist, kuglig zusammenzogen und in dieser Form dauernd verharrten. Ich möchte hier ferner an die Beobachtungen erinnern, die A. Schneider, Brass und O. Zacharias ${ }^{1}$ ) machten, indem sie bei Spermatozoën, Darmepithelzellen und Amoeben ebenfalls nach Zusatz verschiedener Lösungen zum Medium die Form der Pseudopodien verändert fanden. Alle diese $T$ hats a c hen zeigen, wi e die Form amoeboider Organismendureh die Einwirkung äuserer Factoren in typischer Weise

1) $0 . Z$ acharias: „Experimentelle Untersuchungen über Pseudopodienbildung." Im Biol. Centralblatt 1885. 
Die polare Erregung der lebendigen Substanz durch den constanten Strom. 55

bes timmt wird, und sind geeignet sehweren Zweifel an der Constanz der sogenannten "Amoebenarten" z u erwecken.

Schliesslich sind diese Erscheinungen von tiefergehendem Interesse für die Lehre von der Formbildungsmechanik der Organismen. Das Problem der organischen Formbildung ist nicht ein rein morphologisches, es ist ebenso sehr ein physiologisches Problem, denn die erste Voraussetzung für die Bildung organischer Körperformen, wie wir sie z. B. in der Entwicklung der Organismen vor uns haben, ist das intacte Bestehen des Lebensvorgangs. Formbildung und Lebensworgang sind ebenso untrennbar miteinander verbunden wie etwa Energiewechsel und Lebensvorgang, und so ist anch ein Verständniss fuir die Mechanik der Formbildung ohne Berücksichtigung der wichtigsten physiologischen Verhältnisse nicht möglich. Ich glaube das besonders betonen zu müssen, weil mir scheint, als ob viele von den neueren Vorstellungen über Formbildung und damit zusammenhängende Fragen den Fehler baben, dass sie zu ausschliesslich morphologisch gedacht sind. So finde ich vor allem, dass eben grade dem Fundamental-Vorgang, der allen Lebenserscheinungen zu Grunde liegt, dem Stoffwechsel zu wenig Rechnung getragen worden ist. Längst ist bekanntlich der Physiologie die Vorstellung in Fleisch und Blut ïbergegangen, dass sämmtliche Lebenserscheinungen eines Organismus nur Ausdruck seines Stoffwechsels sind. Insofern daher die Bildung und Veränderung der organischen Körperformen Lebenserscheinungen des Organismus sind, müssen sie auch ebenso wie alle anderen Lebenserscheinungen Functionen seines charakteristischen Stoffwechsels sein. In dieser Beziehung baben die oben mitgetheilten Thatsachen besonderes Interesse. Hier sehen wir an einer Zelle, welche vermöge ihrer Fähigkeit des Formwechsels den Einfluss ihres Stoffwechsels auf die Form leicht und deutlich zum Ausdruck bringen kann, wie jede Einwirkung auf den Stoffwechsel, mag sie durch eine polare Erregung seitens des galvanischen Stromes, oder mag sie durch einen Zutritt chemischer Stoffe von Seiten des Mediums zu Stande kommen, sich gleichzeitig. auch in einer Beeinflussung der Körperform äussert, so dass man im Stande ist, auf Wunsch bestimmte Körperformen zu erzengen. Grade die flüssige Beschaffenheit des Amoebenkörpers gestattet es, dass dieser Zusammenhang besonders deutlich hervortritt. Bleiben 
die einwirkenden Factoren dieselben, so bleibt auch die entsprechende Körperform dauernd bestehen. Fliissige Beschaffenheit oder Stoffwechsel und bestimmte Körperform schliessen sich also nicht nur nicht aus, sondern stehen sogar in einem ursächlichen Zusammenhang miteinander. Dureh den Vergleich mit der Gasflamme und dem Springbrunnen habe ich in meiner "Allgemeinen Physiologie" dieses Verbältniss anschaulicher zu machen gesucht. Auch im übrigen kann ich hier bezüglich der allgemeinen Prinzipien, die bei der Formbildungsmechanik der Zelle zu berücksichtigen sind, auf jene Ausführungen verweisen.

In ihrer Mittheilung "zur Theorie des Galvanotropismus" haben Loeb und Maxwel11) den Versuch gemacht, die Erscheinungen der polaren Erregung bei gewissen Protisten mit dem Pflü ger'schen Gesetz der polaren Erregung des Nerven und Muskels in Einklang zu bringen, indem sie verschiedene Aunahmen zu Hülfe genommen haben, die längst durch die Thatsachen selbst widerlegt sind. Ich bin einigermaassen verwundert gewesen, als ich die betreffenden Ausführungen las und kann mir diesen Versuch der beiden Autoren nur durch die Annahme erklären, dass ihnen die zabllosen Thatsachen, die in den letzten Jahren über die polare Erregung einzelliger Organismen gesammelt worden sind, nicht geniigend bekannt waren, oder dass sie wenigstens selbst nie die wesentlichsten Erscheinungen vor Augen gehabt haben. Loeb und Maxwell meinen, dass bei dem Kühne'schen Versuche am Actinosphaerium der Zerfall des Protoplasmas an der Anode einerseits kein Ausdruck für eine Erregung zu sein braucht, andererseits aber auch als Vergiftungserscheinung aufgefasst werden könnte. Sie ïbersehen dabei vollständig 1) dass der Zerfall selbst gar nicht das Primäre ist, sondern dass sich typische Contractionserseheinungen, bei schwachen Strömen sogar allein ohne darauffolgenden Zerfall an der Anode entwickeln, eine Thatsache, die doch wohl kaum anders als eine Erregungserscheinung genannt werden kann ; 2) dass ich bei meinerWiederholung des $\mathrm{K}$ ü h $\mathrm{n}$ e'schen Versuchs mich unpolarisirbarer Electroden bediente, so dass bei den schwachen Strömen, die zur Anwendung kamen, von einer Vergiftung gar keine Rede sein

1) Jacques Loeb und S. S. Maxwell: "Zur Theorie des Galvanotropismus." In diesem Arch. Bd. 63, 1896. 
kann. Es wäre auch sehr merkwürdig, dass die Vergiftungserscheinungen in dem gleichen Wassertropfen nur immer eintreten, wenn der Strom geschlossen, dagegen stets wieder aufhören sollten, wenn der Strom geöffnet wird. Ferner bezüglich des Galvanotropismus der Infusorien ziehen die beiden Autoren den von Herman n betonten Umstand heran, dass es bei den galvanotropisehen Wirkungen des Stromes „nicht auf die äussere Kathode und Anode des Stromes ankomme, sondern auf die Aus- und Eintrittsstellen desselben an den Protoplasmen der wirksamen Gebilde, auf die sogenannten physiologischen Electroden." So zweifellos diese Bemerkung zutrifft für Objecte, die in allen ihren Theilen gleichartig sind, ebenso zweifellos ist sie unzutreffend für den Infusorienkörper mit seinen Wimperdifferenzirungen. Die Annahme von Loeb und Maxwell, dass die galvanotropische Axeneinstellung dadurch zustandekomme, dass jede Wimper bei gewisser Stellung in eine anelectrotonische und eine katelectrotonische Strecke getheilt werde und dass je nachdem die besonders erregbare Stelle derselben sich im Kat- oder Anelectrotonus befindet, Vermehrung oder Verminderung der Geisselbewegung eintrete, ist bereits durch das thatsächliche Verhalten der Wimpern widerlegt, wie den beiden Autoren ein Blick auf Fig. 9, 10, 13 von L u d l o ff's Arbeit ${ }^{1}$ ) gezeigt hätte, wo die Stellung der Wimpern durchaus den thatsächlichen Verhältnissen entsprechend dargestell t worden ist. Das Verhalten der Wimpern bei Paramaecium zeigt ohne weiteres, dass es bei der galvanotropischen Axeneinstellung nicht auf die directe Erregung der Wimpern von Seiten des Stromes, sondern anf ihre Beeinflussung vom Zellkörper aus ankommt. Der Zellkörper wird durch den Strom in eine anodische und eine kathodische Strecke getheilt und dem entsprechend verhalten sich sämmtliche Wimpern der anodischen Seite übereinstimmend und ebenso wieder im entgegengesetzten Sinne sämmtliche Wimpern der kathodischen Seite, mag das Infusorium eine Lagerichtung dem Strom gegenüber einnehmen, welche es will. Ich wundere mich um so mehr, dass Loeb und Maxwell dieses Verhältniss entgangen ist, als sie ja selbst fiur die galvanotropische Axeneinstellung der Krebse das analoge Verhalten, und zwar hier ohne directe Beobachtung

1) LudI off: "Untersuchungen über den Galvanotropismus". In diesem Arch. Bd. 59. 1895. 
lediglich auf dem Wege des theoretischen Schlusses annehmen, indem sie voraussetzen, dass es beim ganzen Thier nicht auf die polare Erregung der Nerven und Muskeln, sondern auf die der Neurone, und dass es bei den Neuronen nicht auf die Erregung der Protoplasmafortsätze und des Axencylinders, sondern auf die des Ganglienzellkörpers ankomme. Im übrigen geben Loeb und Maxwell nicht den geringsten Anhalt dafür, wie es möglich wäre, sich die Axeneinstellung der Infusorien zu denken unter Beibehaltung der Annahme, dass das Pflüg er'sche Erregungsgesetz anch bei ihnen Geltung habe, wäbrend ja auf der anderen Seite der Mechanismus der Axeneinstellung durch directe Beobachtung des Wimperschlages von Lud loff schon festgestellt ist. Eine einzige eigene Beobachtung der in Betracht kommenden Erscheinungen bei Infusorien, bei marinen Rhizopoden und ror allem der im Vorstehenden geschilderten Verhältnisse bei der grossen A moebe proteus würde sie aber überzengt haben, dass gar nicht daran zu denken ist, das Pflü ger'sche Gesetz von der polaren Erreg'ung des Muskels und Nerven auch auf alle anderen Formen der lebendigen Substanz zu iibertragen, sondern dass eben für verschiedene Formen der lebendigen Substanz sehr verschiedene Gesetze der polaren Erregung existieren.

Vor kurzem hat W. Roux an dieser Stelle (Bd. 63, pag 542) darüber geklagt, dass seine Arbeit "Ueber die morphologische Polarisation von Eiern und Embryonen durch den electrischen Strom" von den Physiologen; unter anderem von Hermann, ganz besonders aber von mir in meiner 3. Mittheilung über „die polare $\mathrm{Er}$ regung der lebendigen Substanz" und in meiner „Allgemeinen Physiologie" nicht die ihr gebührende Bericksichtigung erfahren hat. Roux schreibt: „In Bd. 62 dieses Archives Seite 417 berichtet M. Verworn über meine bezüglichen Versuche in einer Weise, welche den Leser glauben macht, dass dieselben im Grunde nichts den Physiologen Interessirendes enthalten. Da derselbe Autor auch in seinem Buche iiber allgemeine Physiologie dieser Arbeit nicht gedenkt, obschon sie manches Neue, seine eigenen Untersuchungen Ueberholende und neue Gesichtspunkte eröffnende bringt, so nehme ich Veranlassung, direkt den Leserkreis dieses Archives auf dieselbe aufmerksam zu machen, indem ich einige Haupter- 
gebnisse hier reproducire, nachdem ein grösseres dutorreferat im biologischen Centralblatt diese Wirkung nicht gehabt hat. Auch L. Herman $\mathbf{n}$ hat in seinem Jahresberichte für Physiologie (1891) nur den Titel der Abhandlung mitgetheilt."

Mit seiner Behanptung, dass ich den Leser glauben mache, seine Versuche enthielten nichts den Physiologen Interessirendes, stellt Roux den Sachverhalt nicht ganz richtig dar, denn ich habe im Gegentheil hervorgehoben, dass die ,interessanten Versuche in mehr als einer Richtung Beachtung verdienen". Diese Worte lässt Roux fort, indem er die betreffende Stelle citirt, and ebenso unvollständig giebt er meine Ansicht über seine Versuche wieder. Es hat mir, wie meine Worte zeigen, durchaus fern gelegen, den Versuchen Roux's ein Interesse für den Physiologen abzusprechen. Was ich behauptet habe, ist lediglich das, dass sie uns keinen Aufschluss über die polare "Erregung" der lebendigen Substanz durch den constanten Strom liefern. Ich will Roux gern in seinem Urtheil beistimmen, wenn er selbst sagt, dass seine eigene Arbeit meine Untersuchungen überholt hat; an meiner obigen Ansicht aber muss ich trotzdem auch jetzt noch festbalten. Ich vermuthe unsere Differenz liegt darin, dass Roux den physiologisehen Begriff der Erregung wohl nicht ganz richtig aufgefasst hat. Unter Erregung verstehe ich die Hervorrufung oder Steigerung normaler Stoffwechselprozesse und der dadurch bedingten Lebenserscheinungen. Nun muss ich sagen, dass ich auch jetzt noch Bedenken trage, die von $\mathrm{R}$ oux beschriebenen polaren Ver. änderungen an Eizellen als polare Erregungswirkungen des constanten Stromes in diesem Sinne zu deuten. Vermuthlich werden wohl Erregungserscheinungen daran betheiligt sein, aber eine einwandsfreie physiologische Deutung dieser Erscheinungen ist bisher meines Wissens noch nicht gegeben, und auch ich kann eine solche nicht liefern. Dieselbe wird für mich da'durch besonders unsicher gemacht, dass Ro ux zu seinen Versuchen keine unpolarisirbaren Electroden verwendet hat, ein Punkt, den ich schon in meiner Arbeit zur Begründung meiner Ansicht angeführt habe, den aber Ro ux wiederum beim Citiren meiner diesbezïglichen Stelle verschweigt. Ich vermag daher noch nicht einmal mit Sicherheit zu entscheiden, was von den betreffenden Erscheinungen als directe Wirkung des Stromes auf die lebendige Substanz der Eizellen, und was als secundäre Wirkung von Seiten der Polarisationspro- 
ducte der Metallelectroden zu betrachten ist: Alles dies habe ich bereits in der angeführten Arbeit auseinandergesetzt. Ich muss daher sagen, dass Roux's neuerliche Ausführungen meine in der vorigen Mittheilung ausgesprochene Ansicht eigentlich garnicht berührt haben, da sich meine Arbeiten, wie der Titel sagt, ausschliesslich mit polaren Erregungswirkungen des constanten Stromes beschäftigen. Sobald Roux den Nachweis führt, dass die von ihm beobachteten polaren Veränderungen an den Eizellen direkte Erregungswirkungen des constanten Stromes sind, und zeigt, welcher Art die Erregung an der Kathode und an der Anode ist, werden die Ergebnisse auch für die allgemeine Frage nach der polaren Erregung der lebendigen Substanz durch den galvanischen Strom ein grösseres Interesse gewinnen. Betreffs der Frage nach dem Verlauf der Stromfäden gegen einen "Intraelectrolyten" haben dagegen meiner Meinung nach die Erfahrungen Roux's auch für den Physiologen nicht wnerhebliches Interesse, was ich bereits in meiner frïheren Mittheilung angedeutet habe.

Sodann wirft mir Ro ux an derselben Stelle wie auch an anderen Orten in gehässigen Anmerkungen vor, dass ich in meiner ${ }_{n}$ allgemeinen Physiologie" die von ihm ermittelten entwicklungsgeschichtlichen Thatsachen nicht genügend berücksichtigt und zum Theil unrichtig dargestellt habe. Soweit Roux's Angriffe sachlicher Natur sind, möchte ich hier darauf eingehen, um die Sachlage klarzustellen ${ }^{1}$ ). Worum es sich dabei eigentlïh handelt, geht aus einer Anmerkung: in Roux's gesammelten Abhandlungen über Entwicklungsmechanik hervor. Hier sehe ich, dass es hauptsächlich zwei Sätze in meiner "allgemeinen Physiologie" sind, die Roux zu seinen Ausfällen veranlasst haben. Erstens habe ich geschrieben: "gegenüber den Beobachtungen von Roux stellte Hertwig fest, dass auch aus einer einzigen Furchungshälfte der Eizelle noch ganze Embryonen sich entwickeln, ja dass sogar aus einzelnen lebensfähigen Stiucken der Eizelle noch normale Embryonen entstehen." Ich hätte vielleicht noch einschieben können „durch directe Ent-

1) Auf Angriffe zu antworten, die lediglich eine persönliche Gehässigkeit ohne irgend ein sachliches Moment zum Ausdruck bringen, halte ich der Würde einer wissenschaftlichen Arbeit nicht für angemessen und kann ich um so eher unterlassen, als derartige Bemerkungen in dem Urtheil Unbetheiligter auf den Verfasser selbst zurückzufallen pflegen. 
wicklung“. Das war aber für den Leser ziemlich selbstverständlich, denn aus dem Zusammenhang geht klar hervor, dass es sich bier um normale Entwicklung und nicht um Re- oder "Postgenerationen" handelt. Nun besteht aber, wenigstens soviel ich aus Roux's Arbeiten erseben kann, die Differenz zwischen Roux und O. Hertwig darin, dass $\mathrm{R}$ oux angiebt, es entwickelten sich aus einer Eibälfte, die keine Reste der anderen mehr enthält, Hemiembryonen, die sich erst secundär auf dem Wege der Post- oder Regeneration $\mathrm{zu}$ ganzen Individuen vervollständigen können, wälrend Hertwig zeigt, dass auch aus wirklichen Hälften durch normale Entwicklung von vornherein ganze Embryonen entstehen. Ich überlasse es danach dem Leser zu beurtheilen, wie viel Falsches über Roux der oben angefürte Satz von mir enthält. Zweitens fühlt sich Roux verletzt, dass ich seine Entwicklungstheorieen fir im wesentlichen präformistisch halte, wobei mir nichts ferner gelegen hat, als die Absicht Roux zu verletzen. Das Recht meine wissenschaftliche Ansicht auszusprechen, wird mir R oux aber wohl zugestehen und meine Worte machen an der betreffenden Stelle nicht den Anspruch einer Darstellung, sondern einer subjectiven Beurtheilung der verschiedenen Entwicklungstheorieen, wie jeder Leser auf den ersten Blick bemerken wird. Im iibrigen dürfte wohl noch Mancher trotz der gegentheiligen Bethenerungen Roux's zu der gleichen Ansicht gelangen, wenn er

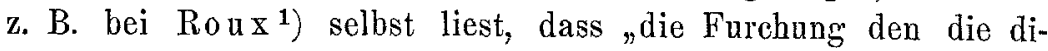
rekte Entwicklung des Individuums vollziehenden Theil des Keimmateriales, insbesondere das Kernmaterial qualitativ seheidet und. mit der dabei stattfindenden Anordnung dieser verschiedenen gesonderten Materialien daher zugleich die Lage der späteren differenzirten Organe des Embryo bestimmt.“ „Für sie gilt His” Princip der organbildenden Keimbezirke, für sie wurde nachgewiesen, dass die Gastrulation eine Mosaikarbeit ist." Derartige Stellen, aus denen ein vorurtheilsfreier Beurtheiler meiner Meinung nach die Vorstellung Roux's für eine modificirt präformistische oder wie ich gesagt habe für „,im Wesentlichen nichts anderes als die alte Präformationslebre in modernerem Gewande" halten mus, kann der Leser zahllos in den Arbeiten Roux's finden.

1) In Virchow's Arch. Bd. 114, 1888, sowie in Verhandl. d. anatom. Gesellsch. in Wien. 1892. 
Ich halte danach die Angriffe Roux's und vor allem die Art, in der sie gemacht werden, durchaus furr ungerechtfertigt und werde in Zukunft nicht wieder darauf eingehen, wenn sich nicht $\mathrm{Roux}$ in seiner Polemik eines rein sachlichen Tones bedient.

\section{Erklärung der Abbildungen anf Tafel IV.}

Fig. 1 und 2. Verschiedene Formen der A moeba proteus in ungestörtem Zustande. Der helle Kreis im körnigen Protoplasma stellt die contractile Vacuole vor. Der Zellkern ist nicht sichtbar. Fig. 1 Polypodiaform, Fig. 2 Wurmform der Amoeba.

Fig. 3. Amoeba proteus vom constanten Strom durchfiossen. An der Anode typische Contraction, an der Kathode Expansion des hyalinen Protoplasmas. Der Pfeil giebt die Kriechrichtung an.

Fig. 4a. Desgleichen.

Fig. 4b. Dasselbe Individuum einige Secunden nach Wendung des Stromes. An der neuen Anode beginnen die Contractionserscheinungen, an der neuen Kathode dringt das Hyaloplasma zwischen und neben den contrahirten Massen vor.

Fig. 5. Hyaline Masse eines Endes durch Umlegen der Wippe zur Anode geworder und contractoriseh erregt. Das hyaline Protoplasma erscheint trübe, vacuolig und an der Oberfläche zerfranzt.

Fig. 6. A moeba proteus im Zustande herabgesetzter Bewegung nach Beendigung längerer Reizversuche. Die Amoebe ist etwas abgeflacht und hat kurze Pseudopodien entwickelt, eine Form, in der sie längere Zeit ohne bemerkenswerthe Bewegung verharrt. 